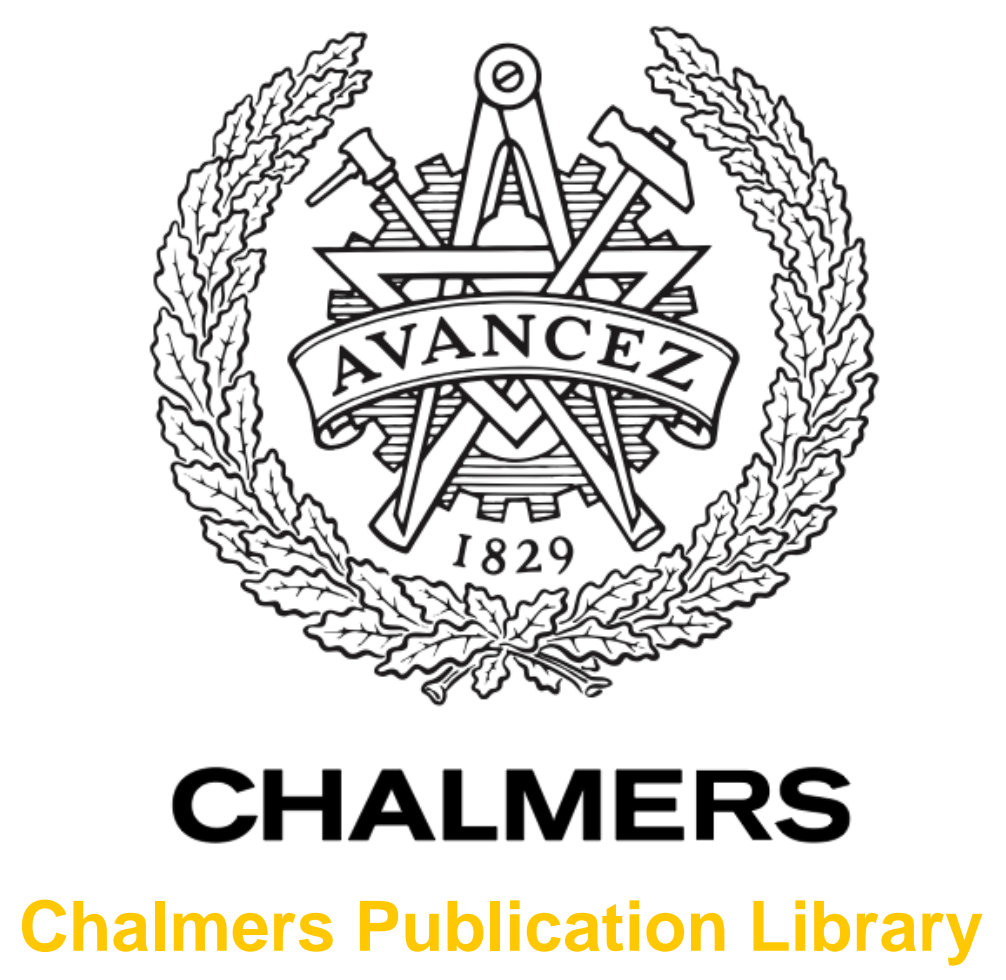

Single parity check multi-core modulation for power efficient spatial super-channels

This document has been downloaded from Chalmers Publication Library (CPL). It is the author's version of a work that was accepted for publication in:

2014 European Conference on Optical Communication, ECOC 2014; Cannes; France; 21 September 2014 through 25 September 2014

Citation for the published paper:

Puttnam, B. ; Mendinueta, J. ; Luís, R. (2014) "Single parity check multi-core modulation for power efficient spatial super-channels". 2014 European Conference on Optical

Communication, ECOC 2014; Cannes; France; 21 September 2014 through 25 September

2014 pp. Art. no. 6964197.

http://dx.doi.org/10.1109/ECOC.2014.6964197

Downloaded from: http://publications.lib.chalmers.se/publication/209236

Notice: Changes introduced as a result of publishing processes such as copy-editing and formatting may not be reflected in this document. For a definitive version of this work, please refer to the published source. Please note that access to the published version might require a subscription.

Chalmers Publication Library (CPL) offers the possibility of retrieving research publications produced at Chalmers University of Technology. It covers all types of publications: articles, dissertations, licentiate theses, masters theses, conference papers, reports etc. Since 2006 it is the official tool for Chalmers official publication statistics. To ensure that Chalmers research results are disseminated as widely as possible, an Open Access Policy has been adopted.

The CPL service is administrated and maintained by Chalmers Library. 


\title{
Single Parity Check Multi-Core Modulation for Power Efficient Spatial Super-channels
}

\author{
B.J.Puttnam ${ }^{1}$, J.-M.Delgado Mendinueta ${ }^{1}$, R.S.Luís ${ }^{1}$, T.A.Eriksson ${ }^{2}$, Y.Awaji ${ }^{1}$, N.Wada ${ }^{1}$ and E.Agrell ${ }^{3}$ \\ (1) Photonic Network System Laboratory, National Institute of Information and Communications \\ Technology (NICT), 4-2-1 Nukui-Kitamachi, Koganei, Tokyo 184-8759, Japan. E-mail:ben@nict.go.jp \\ ${ }^{(2)}$ Department of Microtechnology and Nanoscience, ${ }^{(3)}$ Department of Signals and Systems, \\ Chalmers University of Technology, SE-412 96 Gothenburg, Sweden
}

\begin{abstract}
We investigate multi-core modulation formats for spatial super-channels using a single parity check on PDM-QPSK symbols. Compared to per-core PDM-QPSK, we show improvements in required OSNR of up to $1.8 \mathrm{~dB}$, with minimal impact on spectral efficiency.
\end{abstract}

\section{Introduction}

Recent advancement of space-divisionmultiplexing (SDM) technology, including fibers with up to 19 cores $^{1}$ or 6 spatial modes per polarization ${ }^{2}$, have opened the prospect of using spatial channels to increase the dimensionality of modulation formats. Spatial channels allow multi-dimensional modulation formats that may be compatible with spatial super-channels ${ }^{3}$, recently proposed to simplify switching in SDM networks and used in network demonstrations ${ }^{4}$.

In single core transmission, increasing the dimensionality of the 4-dimensional optical signal space has been investigated by joint modulation of timeslots ${ }^{6,7}$ and adjacent frequency channels ${ }^{5}$. Additionally, applying setpartitioning (SP) to polarization division multiplexed (PDM) formats can improve the asymptotic power efficiency, $\gamma$, at high optical signal-to-noise-ratio (OSNR) for relatively small spectral efficiency (SE) reductions ${ }^{8}$. SP formats use transmission of parity-check bits in each symbol across any number of signal dimensions to reduce the error probability of all transmitted bits. Such formats have been shown to increase transmission reach compared to the PDM-QAM format at identical bit rates ${ }^{9}$.

Here, we investigate single-parity check (SPC) in spatial super-channels, which exploit correlated propagation delay between cores of a multi-core fiber, ${ }^{3,4}$ (MCF). Using a 7-core fiber, we apply this format to QPSK symbols in each quadrature of 3,5 , and 7 cores with one quadrature of one polarization reserved for SPC bit transmission. We show that the sensitivity measured as the required OSNR at a bit error rate (BER) of $10^{-3}$ is improved by up to $1.4 \mathrm{~dB}$ and $1.8 \mathrm{~dB}$ at BER of $10^{-5}$, when compared at the same bit-rate. Furthermore, we show that for high core numbers, almost $3 \mathrm{~dB}$ advantage in $\gamma$ may be obtained with negligible impact on SE.

\section{Single Parity Check Multi-Core QPSK}

The four-dimensional modulation format polarization switched (PS)-QPSK can be described as transmitting independent BPSK symbols in three dimensions, while the fourth is dependent on the other three as an SPC ${ }^{10}$. Hence, it becomes possible to extend this concept to a higher dimensionality by using spatial super-channels transmitted on MCF cores. Using two orthogonal polarization states of $N_{\mathrm{c}}$ fiber cores, independent BPSK symbols are transmitted in $4 N_{\mathrm{c}}-1$ dimensions with the last reserved for the SPC bit, formed as $d_{4 N_{c}}=\operatorname{XOR}\left(d_{1}, d_{2}, \ldots, d_{4 N_{c}-1}\right)$ where $d_{n}$ is the binary sequence in the $n_{\text {th }}$ dimension. The asymptotic power efficiency of this format, referred to as SPC-MC-QPSK, is for the additive white Gaussian noise (AWGN) channel given by

$$
\gamma=\frac{d_{\min }^{2} \log _{2} M}{4 E_{S}}
$$

where $d_{\min }$ is the minimum Euclidean distance ${ }^{10}, M$ is the number of constellation points such that $\log _{2} M=4 N_{c}-1$, and $E_{s}=N_{c}$ is the average symbol energy. The term $d_{\min }$ is increased by a factor of $\sqrt{2}$ compared to PDMQPSK and independent of $N_{c}$, giving an SE of:

$$
S E=\frac{\log _{2} M}{N / 2},
$$

where $N=4 N_{c}$ is the number of dimensions for SPC-MC-QPSK. The SE as a function of $(1 / \gamma)$ for (PDM-)QPSK, PS-QPSK, and SP-MC-QPSK with $N_{c}=1,2, \ldots, 100$ is shown in Fig. 1. SPCMC-QPSK using a single core corresponds to PS-QPSK. Using 2 or more cores, the SE and $\gamma$

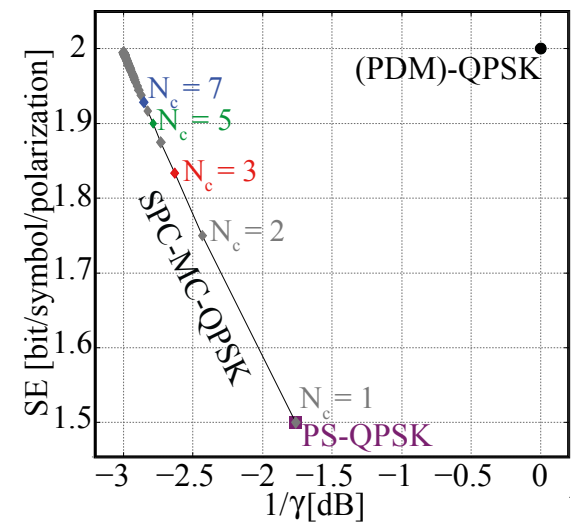

Fig. 1: SE as a function of asymptotic power penalty $(1 / \gamma)$. 


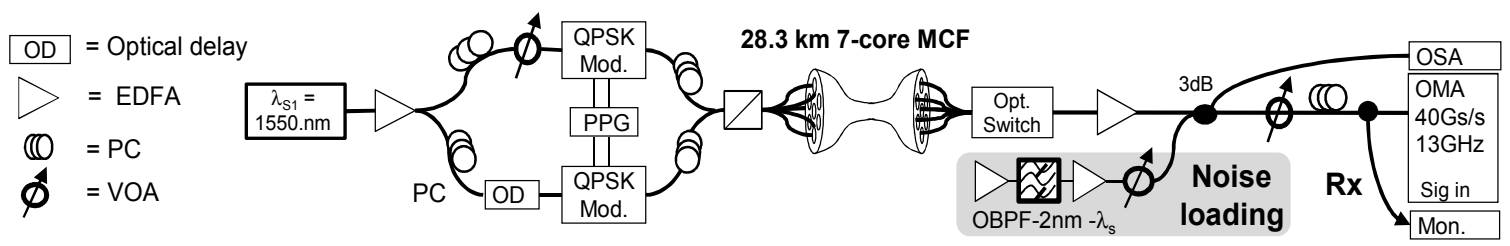

Fig. 2: Experimental Set-up for PDM-QPSK and MC-SP-PDM-QPSK transmission measurements

can be increased simultaneously over PS-QPSK and for an infinite number of cores, SPC-MCQPSK approaches the same SE as QPSK but with $\gamma$ improved by $3 \mathrm{~dB}$.

\section{Experimental Description}

The experimental set-up used for SPC-MCQPSK transmission is shown in Fig. 2. The output of an external cavity laser (ECL) with 700 $\mathrm{kHz}$ linewidth and $1550.5 \mathrm{~nm}$ wavelength was divided in a $3 \mathrm{~dB}$ coupler. In each arm, a dual parallel Mach-Zehnder modulator was used to modulate QPSK signals at a variable baudrate, before recombination on orthogonal polarizations in a polarization beam combiner (PBC). An optical delay (OD) and patch cords were used to align the propagation delay in both paths, with polarization controllers (PCs) used to align the polarization before each modulator and the PBC. A variable optical attenuator (VOA) was used to equalize the power. The generated PDM-QPSK signal was then split between each core of a 7-core MCF of $28.3 \mathrm{~km}$ with delays used to decorrelate signals in each core, with all cores lit for all measurements.

After the fiber, in the absence of 7 coherent receivers, an optical switch was used to select each core in turn for reception. A specific pseudo-random bit sequence (PRBS) provided the signal modulation for each core. For core numbers from 1 to $N_{c}-1$, four decorrelated PRBSs of period $2^{15}-1$ were used for the quadrature of each polarization. For the final core $\left(N_{c}\right)$, three of the bits were generated from additional decorrelated PRBSs, with the quadrature component of the $\mathrm{Y}$ polarization used for transmission of a SPC bit.

Signal reception was performed in a polarization diverse optical coherent receiver connected to a digital sampling oscilloscope with $13 \mathrm{GHz}$ analogue bandwidth. The receiver path also contained an EDFA and a $3 \mathrm{~dB}$ coupler, which was used to add amplified spontaneous emission noise from filtered EDFA outputs to enable BER measurements as a function of the OSNR, with VOAs and optical taps used to control and monitor optical power levels.

For each core, skew, chromatic dispersion compensation, normalization, multiple inputmultiple output (MIMO) polarization tracking, and carrier phase recovery were performed on the traces for each core individually, before the equalized signal structures from all cores were combined for SPC bit processing and error counting. On detection of an SPC bit with odd parity, the received symbol closest to a decision threshold was identified as the most probably error and corrected, before error counting was performed on the reconstructed bit stream with the SPC bit removed. All BER measurements were calculated from the average of three traces, each containing 500,000 symbols. Measurements were performed both at an equivalent symbol rate of $10 \mathrm{GBd}$ and with the clock varied between $9.375 \mathrm{GBd}$ and $10.22 \mathrm{GBd}$ to allow a comparison of PDM-QPSK with 3, 5, and 7 core SPC-MC-QPSK at the same bit-rate.

\section{Results}

Initially, the back-to-back implementation penalty and transmission penalty were measured for each multi-core (MC) format at a fixed symbol rate. Compared to a simulation with AWGN as the only impairment, the implementation penalty for back-to-back transmission was under $0.3 \mathrm{~dB}$, with an additional transmission penalty of $0.2 \mathrm{~dB}$ for all formats. For clarity, the BER as a function of OSNR for the 3 measurements is shown for 7 SPC-MC-QPSK only in Fig. 3.

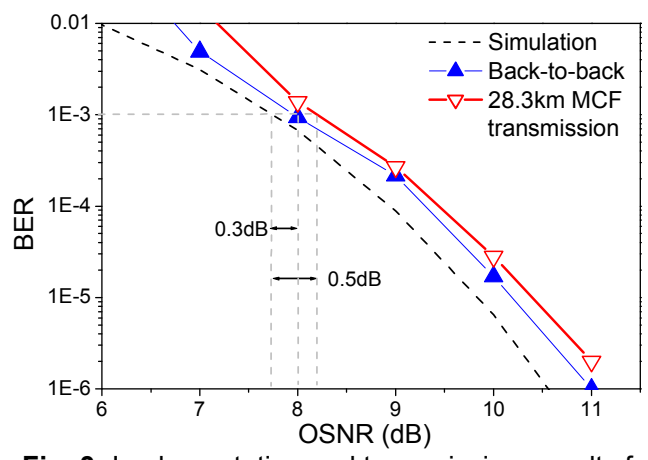

Fig. 3: Implementation and transmission penalty for 7-SPC-MC-QPSK at $10 \mathrm{GBd}$

Fig. 4 shows the average BER plots for the investigated formats after fiber transmission with a fixed symbol rate of $10 \mathrm{GBd}$. Compared to 10 GBd PDM-QPSK, the required OSNR for BER$10^{-3}$ is improved by $1.8,1.3$, and $1.0 \mathrm{~dB}$ for SPCMC-QPSK implemented over 3,5 , and 7 cores, respectively. As may be inferred from the increased $\gamma$ for the MC formats, a larger 


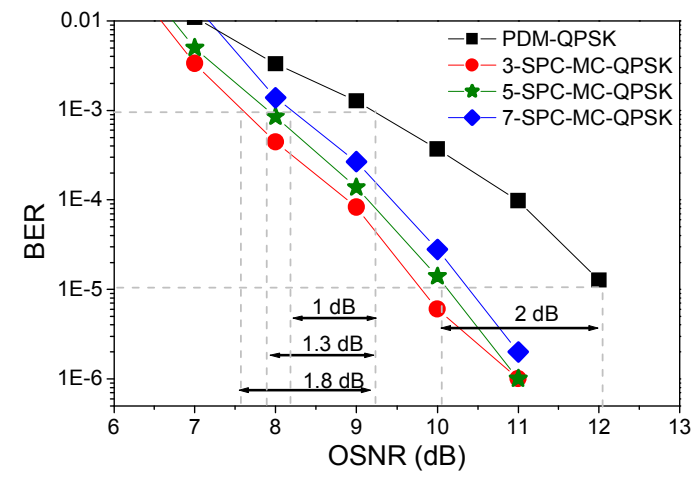

Fig. 4: BER vs OSNR for 1, 3, 5 and 7-SPC-MC-QPSK at fixed symbol rate of $10 \mathrm{GBd}$

sensitivity gain is expected at high OSNR and evidence of this may be seen in the increased required OSNR advantage of the $M C$ formats at BER of $10^{-5}$, which increases to $1.8,2.0$, and 2.2 $\mathrm{dB}$ for 3,5 , and 7 cores, respectively. The higher required OSNR for the higher core number formats may be explained by the fact that the SPC bit is shared over a larger number of data bits. This increases the number of nearest neighbor symbols and, at low OSNRs, increases the probability of multiple errors which may remain uncorrected by the SPC bit.

Finally, the $M C$ formats were compared with PDM-QPSK at the equivalent bit-rate of 37.5 $\mathrm{Gb} / \mathrm{s}$ per core in Fig. 5. This was achieved by varying the clock rate to compensate for the different SEs of the investigated formats. At BER of $10^{-3}$, the spread of the required OSNR between the $M C$ formats reduces to $0.2 \mathrm{~dB}$ as the penalty resulting from increased error probability of sharing the SPC bit between more dimensions is partially compensated by the lower baudrate resulting from the increased spectral efficiency. Compared to PDM-QPSK transmission, at BER of $10^{-3}$, the required OSNR is improved by $1.4,1.3$, and $1.2 \mathrm{~dB}$ for the 3,5 , and 7 core formats with the improvement again increasing to around $1.8 \mathrm{~dB}$ at BER of $10^{-5}$.

Interestingly, although we measure a penalty for increasing the core number at high OSNR, the improved $\gamma$ at higher core numbers should translate to improved sensitivity at even lower BERs. Specifically, $\gamma$ ranges from 2.63 for 3 cores to 2.85 for 7 cores. Hence, although such low BERs cannot be measured for reasonable processing times, a BER threshold should exist where large core numbers become advantageous over smaller ones meaning that the optimum core number depends on the target BER of a specific system which could be an avenue of future study for such formats.

\section{Conclusions}

We have investigated a set of multi-dimensional

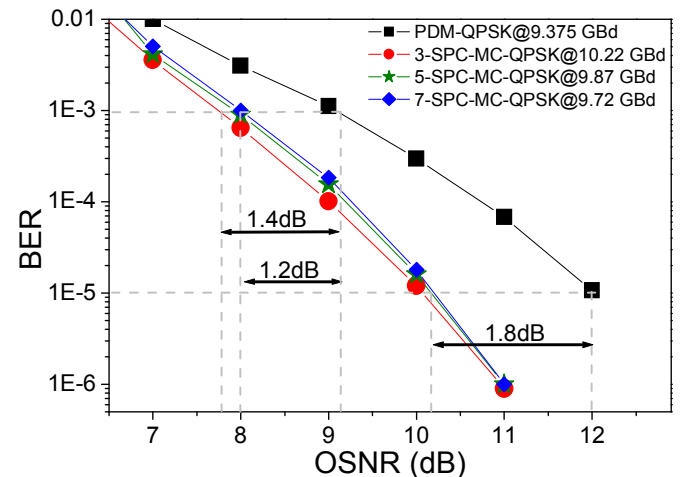

Fig. 5: BER vs OSNR for 1, 3, 5 and 7-SPC-MC-QPSK at equivalent bit-rate of $37.5 \mathrm{~Gb} / \mathrm{s}$ per core

modulation formats suitable for spatial superchannel transmission in multi-core fiber. The formats transmit BPSK in all quadratures of all cores with the exception of a single parity check bit. Theoretical analysis reveals that for high core numbers, the spectral efficiency approaches that of PDM-QPSK, but with an improvement in the asymptotic power efficiency of $3 \mathrm{~dB}$. An experimental investigation in a 7core fiber revealed that the use of this format reduced the required OSNR by up to $1.4 \mathrm{~dB}$ compared to PDM-QPSK at the same bit-rate, with larger improvements at lower BERs. These results not only show that these and similar formats may improve spatial super-channel transmission, but also highlights that adopting multi-core fiber can provide benefits beyond the provision of multiple independent, parallel transmission channels.

\section{References}

[1] J.Sakaguchi et al., "19-core fiber transmission of $19 \times 100 \times 172-G b / s$ SDM-WDM-PDM-QPSK signals at 305 Tb/s ," IEEE JLT 31 (4), 554-562 (2013)

[2] R.Ryf et al., "32-bit/s/Hz Spectral Efficiency WDM Transmission over 177-km Few-Mode Fiber," Proc. OFC/NFOEC, PDP5A.1 (2012)

[3] M.D.Feuer et al.,"ROADM System for Space Division Multiplexing with Spatial Superchannels," Proc. OFC/NFOEC, PDP5B.8 (2013)

[4] N.Amaya et al, "Software defined networking (SDN) over space division multiplexing (SDM) optical networks: Features, benefits and experimental demonstration," Opt. Express 22, 3638-3647 (2014)

[5] T.A.Eriksson et al., "Frequency and Polarization Switched QPSK ," Proc. ECOC Th.2.D.4 (2013)

[6] T.Koike-Akino et al., "Eight-Dimensional Modulation for Coherent Optical Communications," Proc. ECOC Tu.3.C.3, (2013)

[7] T.A.Eriksson, et al, "Biorthogonal Modulation in 8 Dimensions Experimentally Implemented as 2PPM-PSQPSK," Proc. OFC/NFOEC, paper W1A.5 (2013)

[8] G,Ungerboeck, "Channel coding with multilevel/phase signals," IEEE Trans. on Info. Theory, 28, 55-67 (1982)

[9] T.A.Eriksson, et al, "Comparison of 128-SP-QAM and PM-16QAM in long-haul WDM transmission," Optics Express 21 (16), 19269-19279 (2013)

[10]E.Agrell and M.Karlsson, "Power-Efficient Modulation Formats in Coherent Transmission Systems ," IEEE JLT 27 (22) (2009) 